\title{
Concomitant Chemoradiation versus Accelerated Radiotherapy in Selected Cases of Locally Advanced Carcinoma Cervix - A Prospective Study
}

\author{
Abhishek Basu ${ }^{1}$, Kousik Ghosh ${ }^{2}$ Abhijit Basu ${ }^{3}$ \\ ${ }^{I}$ Department of Radiotherapy, R.G. Kar Medical College, Kolkata \\ ${ }^{2}$ Department of Radiotherapy, R.G. Kar Medical College, Kolkata \\ ${ }^{3}$ Department of Radiotherapy, Barasat Cancer Research and Welfare Center, Kolkata
}

\begin{abstract}
Introduction : Although concomitant chemoradiation (CTRT) is the standard of care for locally advanced cervical cancer ( $\mathrm{LACaCx}$ ), it cannot be administered safely in elderly patients or those with certain comorbidities. Aims: To evaluate the feasibility and efficacy of six fractions per week of pure accelerated external beam radiotherapy (EBRT) with standard CTRT, for selected patients with LACaCx. Materials and Methods: From January - September 2010, 45 patients with LACaCx (FIGO stage II B - III B) were administered EBRT 50 Gy / 25 fractions as: 21 in Arm A - Six fractions per week pure accelerated EBRT without chemotherapy and 24 in Arm B - Five fractions per week of conventional CTRT. Patients in both arms received identical brachytherapy. Continuous variables, means-medians were compared with unpaired ' $t$ ' test and categorical data were compared with 'chi square' test. Disease Free Survival (DFS) was assessed using Kaplan Meier curves. Results: Treatment Time was significantly lesser in Arm A ( $p<0.0001)$ with lesser gaps during radiation $(p=0.0156)$. Overall responses were comparable at the end of treatment and last follow up (median 37 months). Acute toxicities were higher in Arm B with significantly lesser average number of adverse events (AEs) in Arm A (All Grades: $p=0.0178$ and Grade $\geq 3: p=0.0314$ ). Late toxicities, 3 year locoregional response and median DFS were similar. Conclusion: The results of our study indicate that pure accelerated EBRT alone may be an effective and feasible alternative to CTRT in certain patients with LACaCx.
\end{abstract}

Keywords: Carcinoma cervix, locally advanced, concomitant chemoradiation, accelerated radiotherapy.

\subsection{Problem}

\section{Introduction}

Carcinoma cervix is the second leading site of primary cancer among Indian women and remains a major health problem.[1-4] Traditionally, a judicious combination of External Beam Radiotherapy (EBRT) and Intracavitary Brachytherapy (ICBT) is the widely accepted primary modality of treatment for carcinoma of the cervix.[5,6]

In spite of advances in radiotherapy, with prospects of enhanced radio-curability in the last few decades, in locally advanced cases, the result of conventionally fractionated primary radiotherapy alone is not very satisfactory.[7]

To circumvent this problem, different investigational procedures were attempted to potentiate radiation but there was no major benefit.[8,9] On the basis of numerous landmark studies in the late 1990s, concomitant chemoradiation with Inj. Cisplatin has become the "standard of care" for treatment of advanced cases of carcinoma cervix at present.[10-14] It was found that cisplatin added to radiation reduces the relative risk of death from cervical carcinoma by decreasing local or pelvic failure and distant metastases.[15] However, cytotoxic chemotherapy cannot be administered safely in elderly patients, those with certain preexisting comorbid medical conditions and in patients who refuse chemotherapy. Therefore, another strategy, without chemotherapy, is required to enhance the effects of radiation in these patients.

\subsection{Previous Work}

Over the years, many studies have shown that prolongation of overall treatment time had detrimental effects on tumour control in carcinoma cervix.[16-21] Overgaard et al have shown that shortening of overall treatment time (keeping total dose constant) improved 5 year locoregional control rates by $10 \%$ in squamous cell carcinomas of the head and neck without significant late toxicities.[22] "As cervical carcinomas have similar tumour characteristics to head and neck cancers"; Yoon SM et al evaluated six versus five fractions of EBRT (followed by ICBT) in a Phase I/II study in cancer cervix patients.[23] They reported that six fractions per week EBRT is an effective treatment for patients of carcinoma cervix and can be used as a possible alternative to concomitant chemoradiation in elderly patients or patients with comorbidity. This approach had the added benefit of avoiding the toxicities associated with chemoradiation. 
Concomitant Chemoradiation versus Accelerated Radiotherapy in Selected Cases of...

\subsection{Purpose and Contribution of the Paper}

Based on the above literature review, we conducted our study in locally advanced carcinoma cervix comparing pure accelerated fractionation alone to concomitant chemoradiation in a selected subset of patients.

The aim of this study was to evaluate the feasibility and compare the efficacy of six fractions per week of accelerated external beam radiotherapy (with conventional fraction size), with standard concomitant chemoradiation, followed by intracavitary brachytherapy for our patients with locally advanced cervix cancer. Treatment intensification by decreasing overall treatment time, by halving the weekend gap, with same dose per fraction was the primary strategy rather than addition of concomitant cytotoxic chemotherapy for selected patients who had contraindication to chemotherapy. Since depriving chemotherapy in ideal circumstances would be suboptimal treatment, the feasibility of an easy to administer altered fractionation schedule as a potential alternative alongwith the proven benefit of reducing treatment time was the main point of focus.

\section{Materials And Methods}

\subsection{Period of Study}

Accrual was done from January 2010 to September 2010.

\subsection{Type of Study}

Prospective, nonrandomized, two arm, single institutional, phase II non inferiority study.

\subsection{Study Population \& Eligibility (Inclusion and Exclusion Criteria)}

All biopsy proven patients of locally advanced squamous cell carcinoma of the uterine cervix (FIGO IIB to IIIB) attending the Out Patients' Department of Medical College \& Hospitals, Kolkata were eligible for this study. All patients had age less than 75 years, ECOG Performance Status $0-2$ and none were pregnant. All patients had a normal baseline hematologic and metabolic profile. None of the patients had previous history of malignancy or exposure to cytotoxic chemotherapy or radiation. Patients undergoing pelvic surgery for their disease were ineligible. None of the patients had clinical or radiological evidence of metastasis at presentation. Patients were also evaluated by cystoscopy and proctoscopy to exclude bladder and/or rectal involvement before enrolment into the study.

Inclusion criteria for those subset of patients who did not receive concomitant chemotherapy (6 fractions per week arm) included age more than 65 years, poor general medical condition or nutritional status, preexisting co-morbid medical conditions (including severe cerebrovascular, cardiovascular or respiratory ailments and uncontrolled diabetes mellitus), serum creatinine clearance $<50 \mathrm{ml} / \mathrm{min}$ (by Cockcroft-Gault formula), serum bilirubin or liver enzymes above upper limit of normal and patient's refusal to receive chemotherapy.

Before starting treatment, all patients were jointly evaluated by Radiation, Gynecological and Medical Oncologists at the Gynecological Tumor Clinic. Informed consent was taken from all patients prior to enrolment. The study design was approved by the Institutional Ethical Committee.

\subsection{Study Design}

Patients were divided into two arms based on above mentioned criteria. In Arm A (Study Arm), patients who had contraindications to concomitant chemoradiation, received 6 (Six) fractions per week of EBRT without chemotherapy from every Monday to Saturday (1 extra fraction of EBRT on Saturdays i.e. Pure Accelerated Radiotherapy). In Arm B (Control / Standard Arm), patients received 5 (Five) fractions per week of radiation from every Monday to Friday. Weekly Injection Cisplatin at the dose of $40 \mathrm{mg} / \mathrm{m}^{2} \mathrm{IV}$ with necessary premedications and adequate hydration was administered on every Monday during external radiation.

EBRT dose of $50 \mathrm{~Gy}$ in 25 fractions using Theratron $780 \mathrm{C}$ Telecobalt machine (Theratronics International Ltd., Canada) was administered through conventional 4 field box technique using CT simulation.

The upper border of the field was usually kept at the L4-L5 interspace and the lower border of the APPA fields were kept at the level of the bottom of the obturator foramina or appropriately lower, if the growth involved the lower third of vagina. The anterior border of the lateral field was placed including the pubic symphysis and the posterior border included the sacrum upto the third sacral vertebra. Midline shielding was not used. Expected treatment time for EBRT in Arm A and Arm B were 29 days and 35 days respectively.

All patients in both arms received High Dose Rate (HDR) Intracavitary Brachytherapy immediately after completion of EBRT. 8 Gy for 3 (consecutive) weekly fractions was given for HDR Brachytherapy using Varian Gammamed Plus Remote Afterloading machine (Varian, Palo Alto, CA, USA) using Ir ${ }^{192}$ isotope with Eclipse Brachyvision software. Because MRI was not routinely used, delineation of High Risk Clinical Target Volume (HRCTV) and Intermediate Risk Clinical Target Volume (IRCTV) were not done as per the Gynaecological GEC ESTRO guidelines [24] and dose was prescribed to point A. The EQD2 aimed was 
approximately $85 \mathrm{~Gy}_{10}$ with dose constraints for bladder and rectum being kept under 90 Gy and 75 Gy EQD2 respectively.

Identical EBRT and ICBT doses and administration procedures ensured proper comparability. Patients were monitored with weekly clinical examination, blood counts and biochemical tests during treatment. Hemoglobin was maintained above $10 \mathrm{gm} / \mathrm{dl}$ throughout treatment, by active intervention (with oral hematinics and / or blood transfusions) if required.

\subsection{Study Endpoints}

The primary endpoint of this study was assessment of Locoregional Response. Secondary endpoints were overall treatment time (OTT), and toxicities (acute and late) and Disease Free Survival. Response was assessed using the Response Assessment in Solid Tumours (RECIST) Criteria version 1.0 at end of EBRT, end of treatment and during follow-up thereafter. Toxicity was reported using the NCI Common Terminology Criteria for Adverse Events (CTCAE) version 4.0.[25]

\subsection{Follow Up}

Patients were followed up by both Radiation and Gynecological Oncologists with detailed physical and gynecological examination and appropriate blood examinations and/or imaging studies. Initially, patients were followed up every month (for the first 6 months), then every 3 months upto 2 years, and 6 monthly thereafter.

\subsection{Statistical Analysis}

Data was assessed using Statistical Package for Social Sciences, (SPSS Inc., Chicago, IL) software version 18.0 and Medcalc version 13.0. For continuous variables, means/medians were compared with unpaired ' $t$ ' test and for categorical set of data, two groups were compared with 'chi square' test. Disease Free Survival was estimated from the date of registration to the date of first occurrence of disease or death or last noted follow up. Survival data were assessed using Kaplan Meier curves. In both situations $95 \%$ Confidence Intervals and $\mathrm{p}$ value $<0.05$ were considered significant. All reported $\mathrm{p}$ values are 2 tailed.

\subsection{Patient Accrual}

\section{Results}

Initially, 60 patients (30 in each arm) were selected for accrual. Of these, 25 patients in arm A and 27 in arm B were actually enrolled after careful scrutiny towards meeting of inclusion and exclusion criteria. 8 patients were excluded -4 because of detection of organ invasion (FIGO stage IV A) and / or metastatic disease during workup, 2 due to refusal to comply with trial protocol / consent related issues and 1 each due to incidental detection of second synchronous primary malignancy and acute myocardial infarction.

Among patients enrolled in Arm A (Study Arm), reasons were : age more than 65 years and general condition inappropriate for treated with concurrent chemoradiotherapy in 5 (25\%), impaired creatinine clearance in $4(16 \%)$, clinically significant cardiac disease (e.g., congestive heart failure, symptomatic coronary artery disease or myocardial infarction within the last 6 months) in $3(12 \%)$, refusal to take chemotherapy in 2 $(8 \%)$, recent cerebrovascular accident, chronic liver disease and other systemic illnesses in 1 each (4\% each) and a combination of these in the rest. However, 2 patients in Arm A and 3 in Arm B failed to complete treatment and were consequently excluded from the study. Reasons included noncompliance with study protocol, refusal to undergo brachytherapy and personal reasons. Ultimately 21 patients in the Study Arm (Arm A) and 24 in Control Arm (Arm B) underwent the study. The median duration of Follow Up of the study was 37 months (range $21-49$ months).

\subsection{Baseline patient characteristics}

Although not a randomized trial, baseline patient characteristics were similar in both arms (Table 1). Majority of the patients in both the arms were of similar age (Median age: Arm A 52 yrs vs Arm B $51.5 \mathrm{yrs} ; \mathrm{p}=$ 0.206), were post or perimenopausal (Arm A 85.71\% vs Arm B 79.16\%; p = 0.85) and most were from poor socioeconomic sections of society (Arm A 52.38\% vs Arm B 50\%; p = 0.88). Women in both arms had borne an average of 3 children and majority had never used any form of contraception. The age at menarche and at last child birth was also comparable between arms. The performance status was 0 in majority of patients (Arm A $61.9 \%$ vs Arm B 66.6\%; $\mathrm{p}=0.98$ ). FIGO stage III B was the most common stage in both groups (Arm A $57.14 \%$ vs $66.66 \%$ Arm $B ; p=0.98)$.

\subsection{Overall treatment time parameters}

The median EBRT time (Table 2) was 30 days in Arm A (Range 29 - 36 days) and 38 days in Arm B (Range $35-44$ days) which was statistically significant ( $p<0.0001 ; 95 \%$ CI 6.35 to 9.15). The median gap in EBRT was 1 day in Arm A (Range $0-7$ days) and 3 days in Arm B (Range $0-9$ days). This was also 
statistically significant $(\mathrm{p}=0.0156 ; 95 \%$ CI 0.35 to 3.15$)$. Delays in treatment were due to acute toxicities $(65.91 \%)$, holidays $(9.09 \%)$, machine breakdown (6.81\%), and miscellaneous other reasons (18.18\%). The median number of concomitant chemotherapy cycles received by patients in Arm B was 5 (Range $4-6$ ). More than $90 \%$ patients received all scheduled cycles per protocol. All patients received 3 fractions of HDR Intracavitary Brachytherapy on three consecutive weeks immediately on completion of EBRT using Manchester system of Intracavitary Applicators. Brachytherapy was started immediately after completing the EBRT to shorten the overall treatment time. Median treatment delay between starting of Brachytherapy and EBRT was 2 days in both arms ( $\mathrm{p}=\mathrm{NS})$. The Overall Treatment Time (OTT) was 55 days in the Study Arm and 61 days in the Control Arm. Although only a 6 day difference between arms, this was statistically significant $(\mathrm{p}<0.0001$; $95 \%$ CI 4.44 to 8.00$)$.

\subsection{Response Assessment}

Per protocol assessment of Response (Table 3) was done at end of EBRT. In Arm A, 9 patients had Complete Response (CR 42.85\%), 10 had Partial Response (47.61\%) and 2 had Stable Disease (SD). In Arm B, 12 patients were in CR (50\%), 9 in PR (37.5\%) and 3 in SD. Although, CRs were higher in Arm B, this was not statistically significant $(\mathrm{p}=0.85)$. When response was ascertained again at the end of treatment, CR remained higher (18 vs 13 patients) in the Concomitant Chemoradiation (Control or Arm B) arm (75\% vs $61.9 \%$ in Arm A) but this did not reach significance $(\mathrm{p}=0.53)$. Moreover, $\mathrm{CR}$ plus PR was comparable between the two groups (90.47\% Arm A vs 91.66\% Arm B; p=0.700).

Overall response $(\mathrm{CR}+\mathrm{PR}+\mathrm{SD})$ at treatment completion was $100 \%$ in both arms. At last follow up, response was again assessed. The response for patients who were lost to follow up was assessed using last recorded data. Complete responses remained comparable among both Arms (42.85\% vs 41.66\% in Arm A vs B; $\mathrm{p}=0.82), \mathrm{CR}+\mathrm{PR}+\mathrm{SD}$ was similar (Arm A 61.9\% vs Arm B 62.5\%; p = 0.87) with 9 and 10 patients in CR in Arms A and B respectively.

Recurrences occurred in both arms. There were 6 recurrences in both arms $(p=N S)$. Arm A had 5 locoregional and 1 distant recurrences compared to 6 in Arm B (all locoregional). The 3 year local control rate was $71.91 \%$ and $75 \%$ in Arms A and B respectively $(\mathrm{p}=0.91)$. The median Disease Free Survival (Fig. 1) was comparable between the arms (42 months in Arm A vs 40 months in Arm B; log rank p value =0.68).

\subsection{Toxicity Assessment}

\subsubsection{Acute Toxicities}

Acute toxicities were assessed using the CTCAE version 4.0 (Table 4). Major toxicities included gastrointestinal, genitourinary, hematological, constitutional and skin related. Grade 3 anorexia, nausea / vomiting and diarrhea were higher in patients receiving chemoradiation $(20.83 \%$ vs $14.2 \%, 25 \%$ vs $4.76 \%$ and $16.67 \%$ vs $9.52 \%$ respectively) but this was not statistically significant. There were more genitourinary toxicities (including bladder spasms, cystitis, incontinence, vaginal pain, mucositis and discharge) in Arm B (46.87\% vs $34.52 \%$; $\mathrm{p}=0.59)$. Among hemato-toxicities of concern, Grade 3 anemia and leucopenia were higher in the concomitant arm $(10.41 \%$ vs $4.76 \%$; $=0.88)$. Fatigue and weight loss were also marginally higher in Arm B. Although dermatological adverse events were higher in Arm B, it was comparable to Arm A ( $p=$ 0.77). When the average number of Adverse Events (AEs) per arm (calculated by dividing the total number of adverse events by the number of patients) was compared, it was found that the higher number of AEs in Arm B was significantly more than in Arm A (12.47 vs 8.82; $\mathrm{p}=0.01)$. Similar results were obtained for the average number of Grade 3 AEs in both arms (Arm B 1.8 vs Arm A 0.75; p =0.0314). There were no Grade 4 adverse events or deaths. All patients completed treatment.

\subsubsection{Late Toxicities}

Late toxicities expectedly were less in number in both the arms. However among the late toxicities that did occur, incidences were similar in both groups $(p=N S)$. Late rectal toxicity was observed in 3 patient in Arm A and 2 in Arm B (both Grade <3; $\mathrm{p}=0.8739$ ). Bladder toxicities were not observed in either arm.

\section{Discussion}

Concomitant chemoradiation is the standard treatment in locally advanced carcinoma cervix and cisplatin appears to be the ideal chemotherapeutic agent.[26] Green et al., analyzed data from 19 randomized trials comprising 4,580 patients and concluded that concomitant chemotherapy results in improved overall survival (RR 0.71; p < 0.0001) and progression-free survival (RR 0.61; p < 0.0001).[27] Although, the absolute survival benefit was $12 \%$, it was maximal in early stage (I and II) disease and patients receiving chemoirradiation had a higher incidence of grade 3 or 4 hematologic and gastrointestinal toxicities. [27] 
Moreover, a recent meta analysis in head and neck cancer has confirmed that the magnitude of the benefit from concomitant chemotherapy is less in older patients.[28]

There is no question about the benefit of chemoradiation in cervical cancer, albeit at the cost of incremental toxicity. However, the best treatment of those patients who cannot tolerate chemoradiation is not very clear. Traditionally, conventional radiation alone has been used in these patients. Considering the fact that radiation alone is a suboptimal treatment in locoregionally advanced cervical cancer, newer avenues to improve local control and perhaps survival should be sought in this group. Many trials have conclusively proven overall treatment time to be a major determinant in outcome in cancer cervix.[16 - 20]

The usual recommendation is to complete treatment by 8 weeks (56 days).[29] Petereit et al. have shown that the 5-year survival and pelvic control rates differed significantly with treatment times $\leq 55$ days vs. $\geq 55$ days: $65 \%$ and 54\% $(\mathrm{p}=0.03)$ and $87 \%$ and $72 \%(\mathrm{p}=0.006)$, respectively.[17] Pure Accelerated Radiotherapy seems a natural choice to circumvent the above two issues. By shortening treatment time, without any alteration of total dose or dose per fraction, treatment can be effectively completed earlier without incremental toxicities usually associated with other altered fractionation schedules like hyperfractionation. This benefit should ideally be extended to those in whom concomitant chemotherapy is not possible, because it gives them tangible benefit over conventional radiation by reducing overall treatment time.

The benefit of Accelerated ( 6 fractions per week) radiation have been conclusively proven in the DAHANCA Trials, where improved local control over conventional 5 days a week radiation were found without toxicities.[22] This theory was extended to cervical cancer and accelerated radiation found to be acceptable in a phase I / II trial.[23]

Over the years, it has been realized that Biological Effective Dose (BED) does not tell the full story since it disregards time, a pivotal element of treatment delivery and hence, modifications to the BED formula incorporating time have been suggested [30]. Considering tumor repopulation at a continuous (exponential) rate throughout treatment, the net effect depends on treatment duration (T) and the effective tumor doubling time $t_{\text {eff. }}$. As a consequence of this, the formula of BED (1) will be as below:

Equation 1: $B E D=n d[1+d / \alpha / \beta]-0.693 / \alpha \cdot t_{\text {eff }}\left(T-T_{k}\right)$

Where $\mathrm{n}$ is the number of fractions, $\mathrm{d}$ is the dose per fraction and $\alpha / \beta$ (often called fractionation sensitivity) is a measure of how a specific tissue will respond to fractionation and dose rate. $\mathrm{T}$ is the Total treatment time and $T_{k}$ is the time from when repopulation starts. The entity $0.693 / \alpha . t_{\text {eff }}$ can be simply expressed as a constant $\mathrm{K}$, the required dose equivalent of repopulation per day. For rapidly proliferating tumors, like cervical cancers, the value of $\mathrm{K}$ is approximately $0.6 \mathrm{~Gy} /$ day (considering $\alpha=0.3 \mathrm{~Gy}^{-1}$ and $\mathrm{t}_{\mathrm{eff}}=3.5-5$ days). There is controversy regarding values of $\mathrm{T}_{\mathrm{k}}$, although 21 days is probably most appropriate, as there is evidence of a time effect for tumor control beyond 3 weeks [30,31]. The simplified formula (2) will therefore be as below:

Equation 2: $\mathrm{BED}=\mathrm{nd}[1+\mathrm{d} / \alpha / \beta]-0.6(\mathrm{~T}-21)$

Data from our trial show that patients in the 6 fractions per week radiation alone arm completed treatment earlier than those in the chemoradiation arm. The treatment gaps were also lesser. Although small in terms of absolute value, the delays in the chemoradiation arm were statistically significant. Considering the fact that patients who were older and had some comorbidities (for which they were denied chemotherapy) were selectively included in the 6 fraction per week arm, the results are definitely encouraging. If we translate this into (1) above, we will find that the BED is $50 \times 1.2$ or 60 Gy in both arms (considering $\alpha / \beta$ value of 10 ). However, if we incorporate the median treatment time (during EBRT in both arms - 30 days in Arm A and 38 days in Arm B), we will get different BEDs of 54.6 Gy $\mathrm{G}_{10}$ in Arm A and 49.8 Gy $\mathrm{G}_{10}$ in Arm B (from (2)). The biologic dose wasted in Arm A is only $5.4 \mathrm{~Gy}_{10}$ compared to almost double of $10.2 \mathrm{~Gy}_{10}$ in Arm B.

In our study, although the shortening of treatment time did not translate into improved local control or reduced recurrence, the results were clearly non-inferior to the standard arm. Complete Response appeared to be relatively higher in the chemotherapy arm, but the difference seemed to be dwindling with time. The long term local control and 3 year disease free survival were similar. Although the patterns of failure were similar, there were distant failures observed in Arm A and none in Arm B. However, too much should not be read into these small numbers.

Another major finding of our study was the increased acute toxicities in the concomitant arm. Both overall and Grade 3 toxicities were consistently higher in the chemotherapy arm. The average number of acute adverse events (both Grade 3 and all grades) i.e. number of adverse events divided by number of patients in each arm was significantly higher in the chemoradiation arm. This is not an unexpected finding, and we found that the prolongation in overall treatment time was due to these adverse events in the majority of patients. In a 
developing country like ours, where delivering treatment under numerous resource constraints is a major challenge, shortening treatment time has other benefits. It facilitates earlier initiation of treatment for more patients by reducing the waiting period and ensures optimization of limited resources.

Although findings from our study vindicate the non-inferiority of accelerated radiation, the results need to be viewed with cautious optimism. This is because our study is plagued by some drawbacks including small sample size, short follow up period and inherent biases of single-institutional trials. Our trial offers an exciting prospect which might be an alternative option in selected patients who have contraindications to chemoradiation and we hope that our results will be validated in larger trials in future to better serve these patients.

\section{Conclusion}

The results of this study suggest that pure accelerated EBRT (six fractions per week) alone followed by HDR brachytherapy is an effective treatment for patients with locally advanced carcinoma of the uterine cervix and can be used as a possible alternative to concomitant chemoradiotherapy in elderly patients or those with certain co-morbidities. The early responses to treatment are non-inferior to concomitant chemotherapy and the acute toxicities lesser. Moreover, overall treatment time, which is of paramount importance for treatment success, is significantly reduced with accelerated 6 fractions per week radiation. This method provides a rational and feasible alternative to conventional chemoradiation in patients of locally advanced cervical cancer who have contraindications to chemotherapy.

However, these findings are not conclusive as a result of the small sample size and the relatively short follow-up period which are limitation of the study. This study generates a hypothesis that, in our view, merits further investigation. Further multicenter, randomized controlled phase III trials will be needed to prove the benefit of the shortening of overall treatment time and comparing the efficacy of accelerated radiotherapy alone vis-a-vis standard chemoradiation.

\section{Acknowledgements}

Since this work was carried out at the Department of Radiotherapy, Medical College \& Hospitals, Kolkata, we would like to acknowledge the help, guidance and support of all the Faculty members, Residents and Staff of the Department at that time.

\section{REFERENCES}

[1]. Indian Council of Medical Research: National Cancer Registry Programme; Consolidated Report of Population Based Cancer Registries 2001 - 2004; incidence and distribution of cancer; December 2006.

[2]. www.ncrpindia.org; www.canceratlasindia.org: websites of National Cancer Registry Programme (Last accessed 12th November, 2014).

[3]. Eifel PJ, Berek JS, Markman MA, Gynecologic Cancers: Cancer of the Cervix, Vagina, and Vulva, in DeVita, Vincent T, Lawrence Theodore S, Rosenberg Steven A. (Eds.) Devita, Hellman \& Rosenberg's Cancer: Principles \& Practice of Oncology, 8 (Lippincott Williams \& Wilkins 2008)

[4]. Sankaranarayanan R, Nene BM, Shastri SS, Jayant K, Muwonge R, Budukh AM, et al, HPV Screening for Cervical Cancer in Rural India. N Engl J Med, 360, 2009, 1385-94.

[5]. Lanciano RM, Won M, Coia LR, Hanks, GE, Pretreatment and treatment factors associated with improved outcome in squamous cell carcinoma of uterine cervix: A final report of the 1973 and 1978 patterns of care studies, Int J Radiat Oncol Biol Phys, 20, 1991, 667-676.

[6]. Perez CA, Breaux S, Madoc-Jones H, Bedwinek JM, Camel HM, Purdy JA, et al. Radiation therapy alone in the treatment of carcinoma of the uterine cervix : Analysis of tumour recurrence, Cancer, 51, 1983, 1393-1402.

[7]. Thoms WW Jr, Eifel PJ, Smith TL, Morris M, Delclos L, Wharton JT, et al. Bulky endocervical carcinoma of the uterine cervix: a 23-year experience, Int J Radiat Oncol Biol Phys, 23, 1992, 491-499.

[8]. Varghese C, Rangad F, Jose CC, Raveendran P, Subhashini J, Ramadas K, et al, Hyperfractionation in advanced carcinoma of the uterine cervix: A preliminary report, Int J Radiat Oncol Biol Phys, 23, 1992, 393-396.

[9]. Rotman M, Pajak TF, Choi K, Clery M, Marcial V, Grigsby PW, et al, Prophylactic extended-field irradiation of para-aortic lymph nodes in stages IIB and bulky IB and IIA cervical carcinomas, Ten-year treatment results of RTOG 79-20. JAMA, 274(5), 1995, 387-93.

[10]. Morris M, Eifel PJ, Lu J, Grigsby PW, Levenback C, Stevens RE, et al, Pelvic radiation with concurrent chemotherapy compared with pelvic and para-aortic radiation for high-risk cervical cancer. N Engl J Med, 340, 1999, 1137-1143.

[11]. Rose PG, Bundy BN, Watkins EB, Thigpen JT, Deppe G, Maiman MA, et al. Concurrent cisplatin-based radiotherapy and chemotherapy for locally advanced cervical cancer, N Eng J Med, 340, 1999, 1144-1153.

[12]. Keys HM, Bundy BN, Stehman FB, Muderspach LI, Chafe WE, Suggs CL 3rd, et al, Cisplatin, radiation, and adjuvant hysterectomy compared with radiation and adjuvant hysterectomy for bulky stage IB cervical carcinoma, N Eng J Med, 340, 1999, 1154-1161.

[13]. Peters WA 3rd, Liu PY, Barrett RJ 2nd, Stock RJ, Monk BJ, Berek JS, et al, Concurrent chemotherapy and pelvic radiation therapy compared with pelvic radiation therapy alone as adjuvant therapy after radical surgery in high-risk early-stage cancer of the cervix, $\mathbf{J}$ Clin Oncol, 18, 2000, 1606-1613.

[14]. Pearcey R, Brundage M, Drouin P, Jeffrey J, Johnston D, Lukka H, et al, Phase III trial comparing radical radiotherapy with and without cisplatin chemotherapy in patients with advanced squamous cell cancer of the cervix, J Clin Oncol, 20, 2002, $966-972$.

[15]. Monk BJ, Tewari KS, Koh WJ. Multimodality therapy for locally advanced cervical carcinoma: state of the art and future directions, J Clin Oncol, 25, 2007, 2952-2965.

[16]. Lanciano RM, Pajak TF, Martz K, Hanks GE, The influence of treatment time on outcome for squamous cell cancer of the uterine cervix treated with radiation: a patterns-of-care study, Int J Radiat Oncol Biol Phys, 25, 1993, 391-397. 
Concomitant Chemoradiation versus Accelerated Radiotherapy in Selected Cases of...

[17]. Petereit DG, Sarkaria JN, Chappell R, Fowler JF, Hartmann TJ, Kinsella TJ, et al, The adverse effects of treatment prolongation in cervical carcinoma, Int J Radiat Oncol Biol Phys, 32, 1995, 1301-1307.

[18]. Girinsky T, Rey A, Roche B, Haie C, Gerbaulet A, Randrianarivello H, et al, Overall treatment time in advanced cervical carcinomas: A critical parameter in treatment outcome, Int J Radiat Oncol Biol Phys, 27, 1993, 1051-1056.

[19]. Erridge SC, Kerr GR, Downing D, Duncan W, Price A, The effect of overall treatment time on the survival and toxicity of radical radiotherapy for cervical carcinoma, Radiother Oncol, 63, 2002, 59-66.

[20]. Gasinska A, Fowler JF, Lind BK, Urbanski K, Influence of overall treatment time and radiobiological parameters on biologically effective doses in cervical cancer patients treated with radiation therapy alone, Acta Oncol, 43, 2004, 657-666.

[21]. Chatani M, Matayoshi Y, Masaki N, Inoue T, High-dose rate intracavitary irradiation for carcinoma of the uterine cervix: the adverse effect of treatment prolongation, Strahlenther Onkol, 173, 1997, 379-384.

[22]. Overgaard J, Hansen HS, Specht L, Overgaard M, Grau C, Andersen E, et al, Five compared with six fractions per week of conventional radiotherapy of squamous-cell carcinoma of head and neck: DAHANCA 6 and 7 randomized controlled trial, Lancet, 362, 2003, 933-940

[23]. Yoon SM, Huh SJ, Park W, Lee JE, Park YJ, Nam HR, et al, Six fractions per week of external beam radiotherapy and High-DoseRate Brachytherapy for carcinoma of the uterine cervix: A phase I/II study, Int J Radiat Oncol Biol Phys, 65, 2006, 1508-1513.

[24]. Haie-Meder Christine, Pötter Richard, Limbergen Erik Van et al, Recommendations from Gynaecological (GYN) GEC ESTRO Working Group (I): concepts and terms in 3D image based 3D treatment planning in cervix cancer brachytherapy with emphasis on MRI assessment of GTV and CTV, Radiother Oncol, 74, 2005, 235-245.

[25]. Cancer Therapy Evaluation Program, Common Terminology Criteria for Adverse Events, Version 4.0, (www.http://ctep.cancer.gov), (Last accessed November 24th, 2014)

[26]. Haie-Meder C, Lhommé C, de Crevoisier R, Morice P, Resbeut M, Concomitant radiochemotherapy in cancer of the cervix uteri: modifications of the standards, Cancer Radiother, 4, 2000, 134S-140S.

[27]. Green JA, Kirwan JM, Tierney JF, Symonds P, Fresco L, Collingwood M, et al, Survival and recurrence after concomitant chemotherapy and radiotherapy for cancer of the uterine cervix: a systemic review and meta-analysis, Lancet 358, 2001, 781-786.

[28]. Pignon JP, le Maître A, Maillard E, Bourhis J; MACH-NC Collaborative Group, Meta-analysis of chemotherapy in head and neck cancer (MACH-NC): An update on 93 randomised trials and 17,346 patients, Radiother Oncol, 92, 2009, 4-14.

[29]. Nag S, Erickson B, Thomadsen B, Orton C, Demanes JD, Petereit D, The American Brachytherapy Society (ABS) Recommendations for High-Dose-Rate Brachytherapy for Carcinoma of the Cervix, Int J Radiat Oncol Biol Phys, 48, 2000, 201211.

[30]. Roberts SA, Hendry JA, The delay before onset of accelerated tumour cell repopulation during radiotherapy: a direct maximumlikelihood analysis of a collection of worldwide tumour-control datasets, Radiother Oncol, 29 1993, 69-74.

[31]. Jones B, Dale RG et al, The potential for mathematical modelling in the assessment of the radiation dose equivalent of cytotoxic chemotherapy given concomitantly with radiotherapy, Br J Radiol, 78, 2005, 939-944.

\section{Tables and Figures}

Table 1: Baseline patient characteristics

\begin{tabular}{|c|c|c|c|c|}
\hline & & $\begin{array}{c}\text { Arm A } \\
(\mathrm{n}=21)\end{array}$ & $\begin{array}{l}\text { Arm B } \\
(\mathrm{n}=24)\end{array}$ & $\mathrm{p}$ value \\
\hline \multirow{3}{*}{ Age (yrs) } & Mean & $\begin{array}{c}53.91 \\
8.40\end{array}$ & $\begin{array}{c}50.96 \\
6.99\end{array}$ & \multirow[t]{3}{*}{$P=0.2059$} \\
\hline & Median & 52 & 51.5 & \\
\hline & Range & $43-72$ & $39-61$ & \\
\hline \multirow{3}{*}{ Weight $(\mathrm{Kg})$} & Mean & 52.38 & 51.71 & \multirow[t]{3}{*}{$P=0.7364$} \\
\hline & Median & 51 & 51.5 & \\
\hline & Range & $42-71$ & $42-65$ & \\
\hline Avg. no. of children & & 3 & 3 & $P=0.1975$ \\
\hline \multirow{2}{*}{ Menopausal status } & Post & $85.71 \%(18)$ & $79.16 \%(19)$ & $\mathrm{P}=0.8552$ \\
\hline & Pre & $14.29 \%(3)$ & $20.84 \%(5)$ & $\mathrm{P}=0.8552$ \\
\hline $\begin{array}{c}\text { Median age at Menarche } \\
\text { (yrs) }\end{array}$ & & $\begin{array}{c}12 \\
(10-15)\end{array}$ & $\begin{array}{c}12 \\
(10-14)\end{array}$ & $P=0.0452$ \\
\hline \multirow{3}{*}{ ECOG P.S. } & 0 & $61.9 \%(13)$ & $66.66 \%(16)$ & $\mathrm{P}=0.9835$ \\
\hline & 1 & $23.8 \%(5)$ & $25 \%(6)$ & $P=0.7993$ \\
\hline & 2 & $14.28 \%(3)$ & $8.33 \%(2)$ & $\mathrm{P}=0.8743$ \\
\hline Poor S.E. Status** & & $52.38 \%(11)$ & $50 \%(12)$ & $\mathrm{P}=0.8890$ \\
\hline $\begin{array}{l}\text { Median Pre Treatment } \mathrm{Hb} \\
(\mathrm{gm} / \mathrm{d})\end{array}$ & & $\begin{array}{c}10.6 \\
(9.3-12.1) \\
\end{array}$ & $\begin{array}{c}10.5 \\
(9.4-12.7) \\
\end{array}$ & $P=0.8042$ \\
\hline Contraceptive Use & & $28.57 \%$ & $25 \%$ & $P=0.9460$ \\
\hline \multirow{3}{*}{ FIGO Stage } & II $\mathrm{B}$ & $28.57 \%(6)$ & $25 \%(6)$ & $P=0.9460$ \\
\hline & III A & $9.52 \%(2)$ & $8.33 \%(2)$ & $\mathrm{P}=0.7001$ \\
\hline & III $B$ & $61.9 \%(13)$ & $66.66 \%(16)$ & $P=0.9835$ \\
\hline
\end{tabular}

Figures in bracket indicate number of patients.

** - Socioeconomic status was determined using the Modified Kuppuswamy's Socioeconomic Scale.

Reference: Bairwa M, Rajput M, and Sachdeva S, Modified Kuppuswamy's Socioeconomic Scale: Social Researcher Should Include Updated Income Criteria, 2012, Indian J Com Med. 38(3), 2013,185-186. 
Concomitant Chemoradiation versus Accelerated Radiotherapy in Selected Cases of...

Table 2: Assessment of Overall Treatment Time (OTT)

\begin{tabular}{|c|c|c|c|c|}
\hline & & $\begin{array}{c}\text { Arm A } \\
(n=21)\end{array}$ & $\begin{array}{c}\text { Arm B } \\
(\mathrm{n}=24)\end{array}$ & $\mathrm{p}$ value \\
\hline \multicolumn{2}{|c|}{ Median EBRT Time } & 30 days & 38 days & $\mathrm{P}<0.0001$ \\
\hline \multicolumn{2}{|c|}{ Median Gaps during EBRT } & 1 day & 3 days & $\mathrm{P}=0.0156$ \\
\hline \multicolumn{2}{|c|}{ Median OTT } & 55 days & 61 days & $\mathrm{P}<0.0001$ \\
\hline \multirow{2}{*}{ Follow up } & Median & 37 months & 37 months & \multirow{2}{*}{ NS } \\
\hline & Range & $21-46$ months & $23-49$ months & \\
\hline
\end{tabular}

Table 3: Assessment of Response to Treatment (as per RECIST criteria)

\begin{tabular}{|c|c|c|c|c|}
\hline & & $\begin{array}{c}\text { Arm A } \\
(\mathrm{n}=21)\end{array}$ & $\begin{array}{c}\text { Arm B } \\
(\mathrm{n}=24)\end{array}$ & $\mathrm{p}$ value \\
\hline \multirow{2}{*}{$\begin{array}{c}\text { End } \\
\text { of } \\
\text { Treatment }\end{array}$} & $\mathrm{CR}$ & $61.9 \%(13)$ & $75 \%(18)$ & $\mathrm{P}=0.5327$ \\
\cline { 2 - 5 } & $\mathrm{CR}+\mathrm{PR}$ & $90.47 \%(19)$ & $91.66 \%(22)$ & $\mathrm{P}=0.7002$ \\
\hline $\begin{array}{c}\text { At } \\
\text { last } \\
\text { Follow } \\
\text { up }\end{array}$ & $\mathrm{CR}$ & $42.85 \%(9)$ & $41.66 \%(10)$ & $\mathrm{P}=0.8244$ \\
\cline { 2 - 5 } & $\mathrm{CR}+\mathrm{PR}$ & $61.9 \%(13)$ & $62.5 \%(15)$ & $\mathrm{P}=0.7891$ \\
\hline
\end{tabular}

CR-Complete Response, PR -Partial Response. Figures in bracket indicate number of patients.

Table 4: Assessment of toxicities (as per CTCAE criteria)

\begin{tabular}{|c|c|c|c|c|}
\hline \multicolumn{5}{|c|}{ Acute toxicities } \\
\hline & & $\begin{array}{c}\text { Arm A } \\
(\mathrm{n}=21)\end{array}$ & $\begin{array}{c}\text { Arm B } \\
(\mathrm{n}=24)\end{array}$ & $\mathrm{p}$ value \\
\hline \multirow[t]{2}{*}{ Gastrointestinal } & All & $58.09 \%(12)$ & $65.83 \%(16)$ & $\mathrm{P}=0.8211$ \\
\hline & Grade 3 & $6.66 \%$ & $15 \%$ & $\mathrm{P}=0.6798$ \\
\hline GenitoUrinary & All & $34.52 \%$ & $46.87 \%$ & $\mathrm{P}=0.5917$ \\
\hline \multirow[t]{2}{*}{ Hematological } & All & $40.47 \%$ & $50 \%$ & $P=0.7335$ \\
\hline & Grade 3 & $4.76 \%$ & $10.41 \%$ & $\mathrm{P}=0.8822$ \\
\hline \multirow[t]{2}{*}{ Constitutional } & All & $34.92 \%$ & $45.83 \%$ & $P=0.6606$ \\
\hline & Grade 3 & $1.58 \%$ & $5.55 \%$ & $\mathrm{P}=0.9301$ \\
\hline Skin / Sub Cutaneous tissue & All & $19.04 \%$ & $27.08 \%$ & $\mathrm{P}=0.7772$ \\
\hline Avg. no. of AE s (All) & & 8.82 & 12.47 & $\mathrm{P}=0.0178$ \\
\hline Avg. no. of AE s (G3) & & 0.75 & 1.8 & $P=0.0314$ \\
\hline \multicolumn{5}{|c|}{ Late toxicities } \\
\hline Late Rectal & & $14.28 \%(3)$ & $8.33 \%(2)$ & $\mathrm{P}=0.8739$ \\
\hline Late bladder & & 0 & 0 & - \\
\hline
\end{tabular}

Figure 1: Kaplan Meir Curve for Disease Free Survival

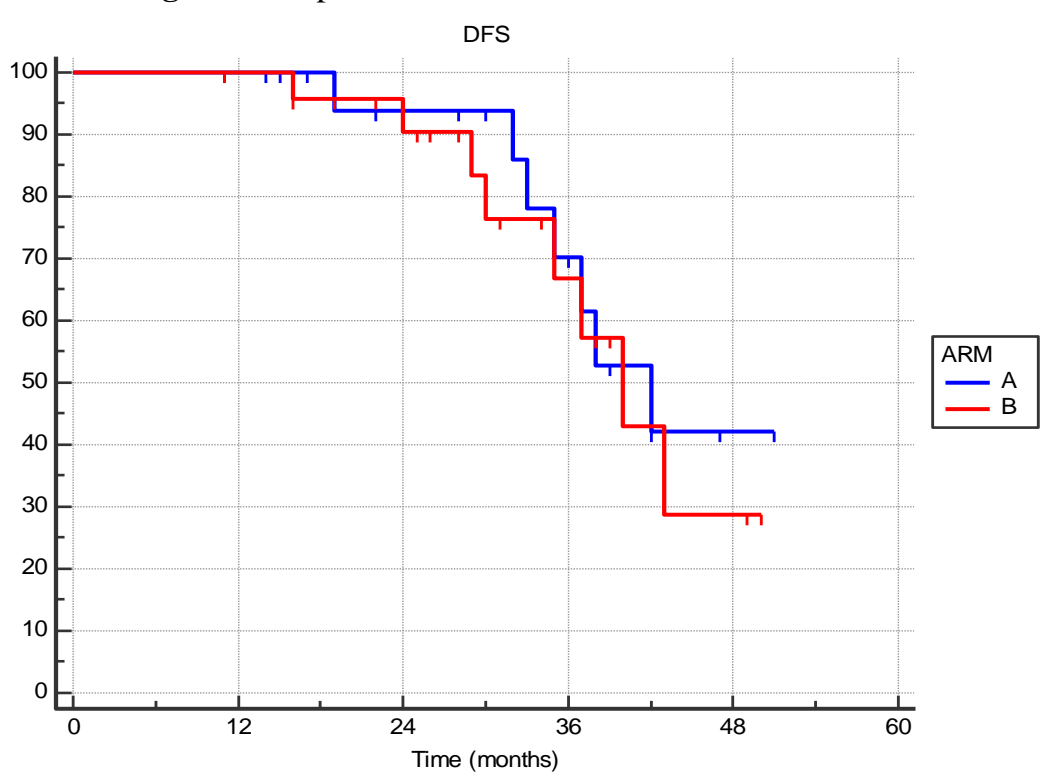

Median Survival: Arm A - 42 months (95\% CI 35 - 42 months); Arm B - 40 months (95\% CI $35-43$ months) Logrank p value $=0.6823$. 